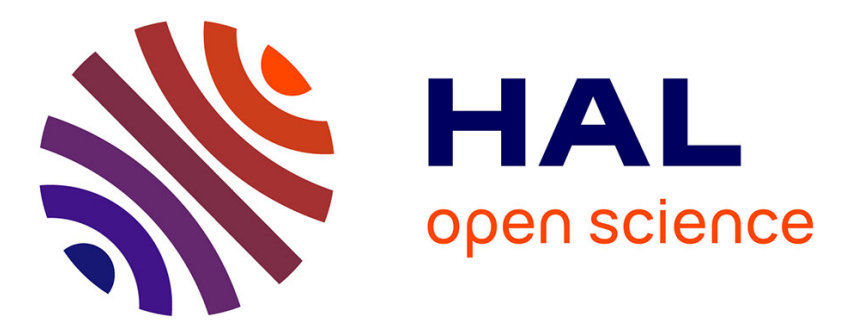

\title{
Preparation of Polylactide/Graphene Composites From Liquid-Phase Exfoliated Graphite Sheets
}

\author{
Xianye Li, Yinghong Xiao, Anne Bergeret, Marc Longerey, Jianfei Che
}

\section{To cite this version:}

Xianye Li, Yinghong Xiao, Anne Bergeret, Marc Longerey, Jianfei Che. Preparation of Polylactide/Graphene Composites From Liquid-Phase Exfoliated Graphite Sheets. Polymer Composites, 2014, 35 (2), pp.396-403. 10.1002/pc.22673 . hal-02914361

\section{HAL Id: hal-02914361 \\ https://hal.science/hal-02914361}

Submitted on 25 May 2021

HAL is a multi-disciplinary open access archive for the deposit and dissemination of scientific research documents, whether they are published or not. The documents may come from teaching and research institutions in France or abroad, or from public or private research centers.
L'archive ouverte pluridisciplinaire HAL, est destinée au dépôt et à la diffusion de documents scientifiques de niveau recherche, publiés ou non, émanant des établissements d'enseignement et de recherche français ou étrangers, des laboratoires publics ou privés. 


\title{
Preparation of Polylactide/Graphene Composites From Liquid-Phase Exfoliated Graphite Sheets
}

\author{
Xianye Li, ${ }^{1}$ Yinghong Xiao, ${ }^{2}$ Anne Bergeret, ${ }^{3}$ Marc Longerey, ${ }^{3}$ Jianfei Che ${ }^{1}$ \\ ${ }^{1}$ Key Laboratory of Soft Chemistry and Functional Materials, Nanjing University of Science and Technology, \\ Nanjing 210094, China \\ ${ }^{2}$ Jiangsu Collaborative Innovation Center of Biomedical Functional Materials, Jiangsu Key Laboratory of \\ Biomedical Materials, College of Chemistry and Materials Science, Nanjing Normal University, \\ Nanjing 210046, China \\ ${ }^{3}$ Materials Center, Ales School of Mines, 30319 Ales Cedex, France
}

\begin{abstract}
Polylactide (PLA)/graphene nanocomposites were prepared by a facile and low-cost method of solutionblending of PLA with liquid-phase exfoliated graphene using chloroform as a mutual solvent. Transmission electron microscopy (TEM) was used to observe the structure and morphology of the exfoliated graphene. The dispersion of graphene in PLA matrix was examined by scanning electron microscope, X-ray diffraction, and TEM. FTIR spectrum and the relatively low $I_{\mathrm{D}} / I_{\mathrm{G}}$ ratio in Raman spectroscopy indicate that the structure of graphene sheets (GSs) is intact and can act as good reinforcement fillers in PLA matrix. Thermogravimetric analysis and dynamic mechanical analysis reveal that the addition of GSs greatly improves the thermal stability of PLA/GSs nanocomposites. Moreover, tensile strength of PLA/GSs nanocomposites is much higher than that of PLA homopolymer, increasing from 36.64 (pure PLA) up to 51.14 MPa (PLA/GSs-1.0).
\end{abstract}

\section{INTRODUCTION}

Polylactide (PLA), a renewable, sustainable, biodegradable, and eco-friendly thermoplastic polyester, has balanced properties of mechanical strength [1], thermal plasticity [2], and compostibility for short-term commod-

Correspondence to: Y.H. Xiao; e-mail: yhxiao@njnu.edu.cn or J.F. Che; e-mail: xiaoche@mail.njust.edu.cn

Contract grant sponsor: Specialized Research Fund for the Doctoral Program of Higher Education of China; contract grant number: 20123219110010; contract grant sponsor: Natural Science Foundation of Jiangsu Province of China; contract grant number: BK2012845; contract grant sponsors: Priority Academic Program Development of Jiangsu Higher Education Institutions (PAPD), contract grant sponsor: Financial support for short visit from Ales School of Mines, France. ity applications [3, 4]. It is currently considered as a promising polymer for various end-use applications for disposable and degradable plastic products [5-8]. Nevertheless, improvement in thermal and mechanical properties of PLA is still needed to pursue commercial success. To achieve high performance of PLA, many studies on PLA-based nanocomposites have been performed by incorporating nanoparticles, such as clays [9, 10], carbon nanotubes [11-13], and hydroxyapatite [14]. However, research on PLA-based nanocomposites containing graphene sheets (GSs) or graphite nanoplatelets has just started [15-17]. GSs exhibit unique structural features and physical properties. It has been known that GSs have excellent mechanical strength (Young's modulus of 1,060 GPa) [18], electrical conductivity of $10^{4} \mathrm{~S} / \mathrm{cm}$ [19], high specific surface area of $2,630 \mathrm{~m}^{2} / \mathrm{g}$ [20], and thermal stability [21]. Polymer nanocomposites based on graphene show substantial property enhancement at much lower filler loadings than polymer composites with conventional micron-scale fillers, such as glass [22] or carbon fibers [23], which ultimately results in lower filler ratio and simple processing. Moreover, the multifunctional property enhancement of nanocomposites may create new applications of polymers.

However, the incorporation of graphene into PLA matrix is restricted by cost and yield. Although the weak interactions that hold GSs together in graphite allow them to slide readily over each other, the numerous weak bonds make it difficult to separate GSs homogeneously in solvents and polymer matrices [24]. Many methods have been reported for exfoliation of graphite, such as intercalation with alkali metals [25] or oxidation in strong acidic conditions [26-29]. Recently, exfoliation of graphite in liquid-phase was found to be able to give oxide-free GSs with high quality and yield at relatively low cost [30-35]. 
This approach is direct, simple, and benign with no need of toxic chemicals and GSs can be dispersed at concentrations of up to $17 \mathrm{mg} / \mathrm{mL}$ in some high boiling point solvents [31], and $0.5 \mathrm{mg} / \mathrm{mL}$ in some low boiling point solvents [32].

Here we report a new, facile, and low-cost method to prepare PLA/GSs nanocomposite materials with high thermal and mechanical performance. In particular, GSs were prepared via liquid-phase exfoliation in chloroform. After solution-blending with PLA, GSs dispersion can be readily turned into nanocomposites. Compared to traditional exfoliation media, such as dimethylformamide (DMF) or $N$-methyl-2-pyrrolidone (NMP), chloroform has obvious advantages of being eco-friendly due to its low toxicity and is easy to get rid of from PLA/GSs blend, thanks to its low boiling point. Graphene oxide (GO) with more oxide groups may be another candidate filler for PLA. However, the preparation process of GO is more complex and the resulting GO is hard to disperse in organic solvents. Moreover, some dangerous reagents, such as sulfuric acid, may be used in GO preparation. Our approach presented here is direct, simple, and benign with no need of toxic chemicals. The liquid-phase exfoliated graphene is a promising candidate of nanocomposite fillers in practical applications. Morphology, structures, and thermal and mechanical properties of PLA/GSs were systematically studied and characterized. With GSs dispersing homogeneously in the matrix, the thermal stability of PLA is improved tremendously. $E^{\prime}$ of the resulting PLA/ GSs increases from 6.87 for pure PLA to $212.15 \mathrm{MPa}$ for PLA/GSs- 1.0 at $100^{\circ} \mathrm{C}$, about 30 orders of magnitude higher compared to that of the unreinforced matrix.

\section{EXPERIMENTAL}

\section{Materials}

Graphite powder (1-44 $\mu \mathrm{m})$ was provided by Sinopharm Chemical Reagent Co., Ltd. PLA 4032D (solid particles) was purchased from NatureWorks ${ }^{\circledR}$, USA, which can be converted into a biaxially oriented film with excellent optical properties, good machinability, and excellent twist and dead fold. Other reagents were purchased from commercial suppliers (Lingfeng Co. Shanghai) and used without further purification.

\section{Preparation of GSs}

Graphite powder was dispersed in chloroform by sonication for $30 \mathrm{~min}(300 \mathrm{~W}, 2 \mathrm{~s}$ on/2 s off $)$ with a tip sonication instrument (Scientz-IID, Ningbo Scientz Biotechnology Co., Ltd.). The resulting dispersion was centrifuged at $1000 \mathrm{rpm}$ for $15 \mathrm{~min}$ (TGL-16G, Shanghai Yuefeng Instruments \& Meters Co., Ltd). After centrifugation, the top $80 \%$ of the supernatant of liquid-phase exfoliated GSs was decanted and collected for the following use.

\section{Preparation of PLA/GSs Nanocomposites}

PLA/GSs nanocomposites were prepared through a solution-blending method, in which chloroform was used as a mutual solvent at different GSs loadings. PLA was completely dissolved in chloroform at a concentration of $100 \mathrm{mg} /$ $\mathrm{mL}$ at $45^{\circ} \mathrm{C}$. The PLA solution was subsequently mixed with GSs dispersion at a certain ratio and stirred for $4 \mathrm{~h}$ at $40^{\circ} \mathrm{C}$ to obtain the homogeneous PLA/GSs solution. After sonication for another $10 \mathrm{~min}$, the mixed solution was poured into a Polytetrafluoroethylene (PTFE) mold and the solvent was evaporated at room temperature to obtain PLA/GSs nanocomposite film with size of about $80 \times 70 \times 0.50 \mathrm{~mm}^{3}$. Finally, the film was dried at $60^{\circ} \mathrm{C}$ under vacuum conditions for 4 days to remove the residual solvent completely. For comparison, PLA was mixed with GS of $0.01,0.1,0.2,0.51 .0$, and $2.0 \mathrm{wt} \%$, respectively. The products are abbreviated as GSs0.01, GSs-0.1, GSs-0.2, GSs-0.5, GSs-1.0, and GSs-2.0.

\section{Characterization}

IR spectrum was recorded using a Shimadzu FTIR8400S spectrometer with pure $\mathrm{KBr}$ as the background. Raman spectra were taken on a Renishaw RM2000, using a $\times 100$ objective lens with a $514.5 \mathrm{~nm}$ laser excitation. $\mathrm{X}$-ray powder diffraction (XRD) analyses were carried out on a Bruker D8 Advance diffractometer at $40 \mathrm{kV}$ and 40 $\mathrm{mA}$ with $\mathrm{Cu} \mathrm{Ka}$ radiation $(\lambda=0.15418 \mathrm{~nm})$, and the diffraction data were recorded for $2 \theta$ angles between $16^{\circ}$ and $19^{\circ}$. Scanning electron microscopy (SEM) observations were performed on a JEOL S-4800. The samples were coated with a thin layer of gold prior to SEM examination. Transmission electron microscopy (TEM) images of graphene and nanocomposite were obtained using a JEOL 2100 , operated at $200 \mathrm{kV}$. TEM samples of composites were cryo-microtomed (Leica Ultracut) with a glass knife into $\sim 100 \mathrm{~nm}$ thick slices and placed on 400 mesh $\mathrm{Cu}$ TEM grids. The composites were embedded in an epoxy matrix (TRA-BOND 2115, Tra-Con) prior to the microtome. Bright field TEM micrographs were obtained with JEOL 2100 at an accelerating voltage of $120 \mathrm{kV}$. Thermogravimetric analysis (TGA) was performed using Shimadzu DTG-60. Samples of neat PLA and PLA/GSs nanocomposites were heated to $600^{\circ} \mathrm{C}$ from room temperature at a rate of $10^{\circ} \mathrm{C} / \mathrm{min}$ in nitrogen atmosphere. The nitrogen flow rate was held at $20 \mathrm{~mL} / \mathrm{min}$. Dynamic mechanical analysis (DMA) was performed on a Metravib DMA-50 dynamic mechanical analyzer (France). The average sample size was $25 \times 4 \times 0.7 \mathrm{~mm}^{3}$ and the measurements were taken in tensile mode from 0 to $140^{\circ} \mathrm{C}$ at a heating rate of $3^{\circ} \mathrm{C} / \mathrm{min}$ and frequency of $1 \mathrm{~Hz}$. The tensile tests were carried out on an Instron 1121 machine at room temperature with a crosshead speed of $50 \mathrm{~mm} / \mathrm{min}$.

\section{RESULTS AND DISCUSSIONS}

A liquid-phase exfoliation method was applied to exfoliate graphite powder in chloroform to obtain GSs. By 

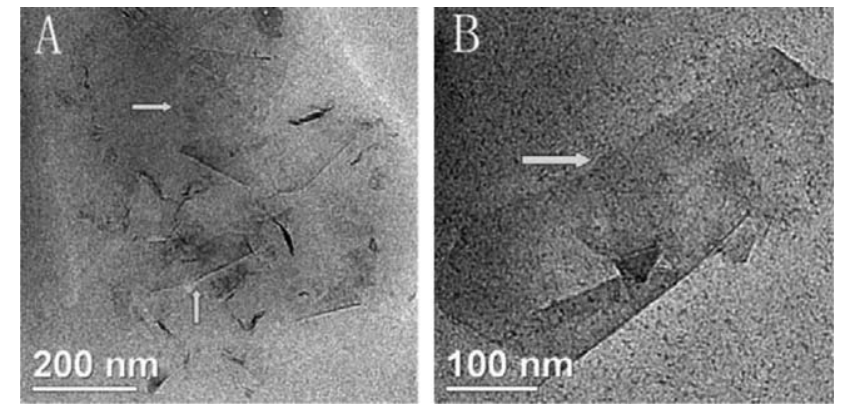

FIG. 1. TEM images of GSs deposited from GS dispersion. (A) An aggregate of GSs and (B) a single layer of GS.

using this benign method, complicated oxidation-reduction process and hazardous chemicals were avoided. After centrifugation and removal of the precipitation, a lightgray GSs dispersion with concentration as high as 0.4581 $\mathrm{mg} / \mathrm{mL}$ was obtained, which could remain stable for over a month. It is known that the typical color of GO dispersion is brown-yellow. The distinct contrast between the colors suggests that there is nearly no oxidation in the process of exfoliation. TEM was applied to characterize the dispersion after centrifugation and it was found that almost all the sheets observed were flat and transparent (Fig. 1A and B), indicating a mono- and few-layer structure of GSs.

The quality of graphene, mainly determined by the content of oxygen functional groups and structural defects, plays a crucial role in the final properties of GSs. From FTIR spectra shown in Fig. 2A we see that unlike traditional GO, the as-made GSs show no absorbances for the $\mathrm{C}=\mathrm{O},-\mathrm{COOH}$, and $\mathrm{C}-\mathrm{O}$ groups, of which characteristic features are often observed around 1,700 and $1,000 \mathrm{~cm}^{-1}$. Raman spectrum for GSs is shown in Fig. $2 \mathrm{~B}$, with the spectra of graphite powder and chemically reduced graphenes (CRGs) as comparison. Three prominent bands can be seen: D band, G band, and 2D band appearing around $1,350,1,580$, and $2,700 \mathrm{~cm}^{-1}$, respectively. The band frequencies and intensity ratios of $\mathrm{D}$ and $\mathrm{G}$ bands are listed in Table 1. It is well known that the intensity ratio of $\mathrm{D}\left(I_{\mathrm{D}}\right)$ to $\mathrm{G}\left(I_{\mathrm{G}}\right)$ band can be used as an indicator of defect quantity. The fewer the defects, the lower the $I_{\mathrm{D}} / I_{\mathrm{G}}$ value. As for graphene, these defects are generally attributed to basal plane defects and edge defects. After sonication, GSs are cut into smaller pieces and a number of new edges form, and thus $I_{\mathrm{D}} / I_{\mathrm{G}}$ of the as-prepared GSs is higher than that of graphite powder. However, it is still much lower than that of CRGs, indicating that the structure of GSs is well preserved during liquid-phase exfoliation.

To investigate the dispersibility of GSs in PLA matrix, SEM and TEM observations were performed. The SEM images of the fractured sections of PLA/GSs-0.1, PLA/ GSs-1.0, and PLA/GSs-2.0 and the TEM images of PLA/ GSs-0.1 are shown in Fig. 3. SEM images show that GSs are dispersed homogeneously throughout the surfaces without any obvious large-size aggregates. The homoge-
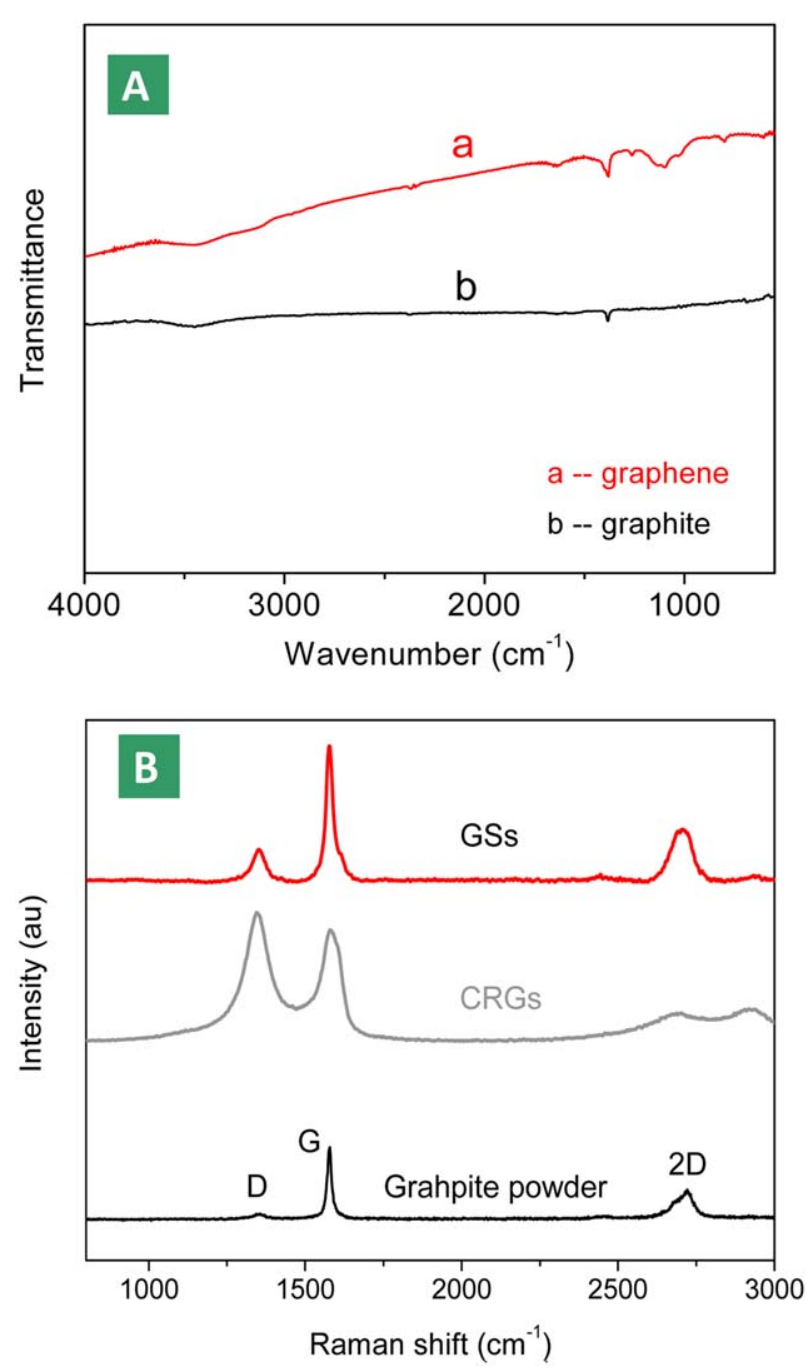

FIG. 2. (A) FTIR spectra of GSs (a) and graphite powder (b). (B) Raman spectra for graphite powder, CRGs, and GSs. [Color figure can be viewed in the online issue, which is available at wileyonlinelibrary.com.]

neous dispersing may be determined by two factors: (1) GS dispersion can maintain the stability during solution blending and (2) PLA macromolecules between GSs prevent the nano-sheets from aggregating and re-stacking during solvent evaporation. The cryofractured surfaces of composites with 0.1 and $1.0 \mathrm{wt} \%$ GSs (Fig. 3A and B) are very dense and no GSs is observed to be pulled out from the surface, suggesting strong PLA-GSs interfacial adhesion. With increase of the GS content (2.0 wt $\%$, Fig. 3C), there are more GSs "competing" for the matrix,

TABLE 1. Frequency of D, G and $2 \mathrm{D}$ bands and intensity ratio of $\mathrm{D}$ and $\mathrm{G}$ bands.

\begin{tabular}{lcccc}
\hline Sample & $\omega_{\mathrm{D}}\left(\mathrm{cm}^{-1}\right)$ & $\omega_{\mathrm{G}}\left(\mathrm{cm}^{-1}\right)$ & $\omega_{2 \mathrm{D}}\left(\mathrm{cm}^{-1}\right)$ & $I_{\mathrm{D}} / I_{\mathrm{G}}$ \\
\hline Graphite & 1357 & 1579 & 2722 & 0.08 \\
CRGs & 1345 & 1581 & 2683,2925 & 1.28 \\
GSs & 1352 & 1576 & 2705 & 0.41 \\
\hline
\end{tabular}



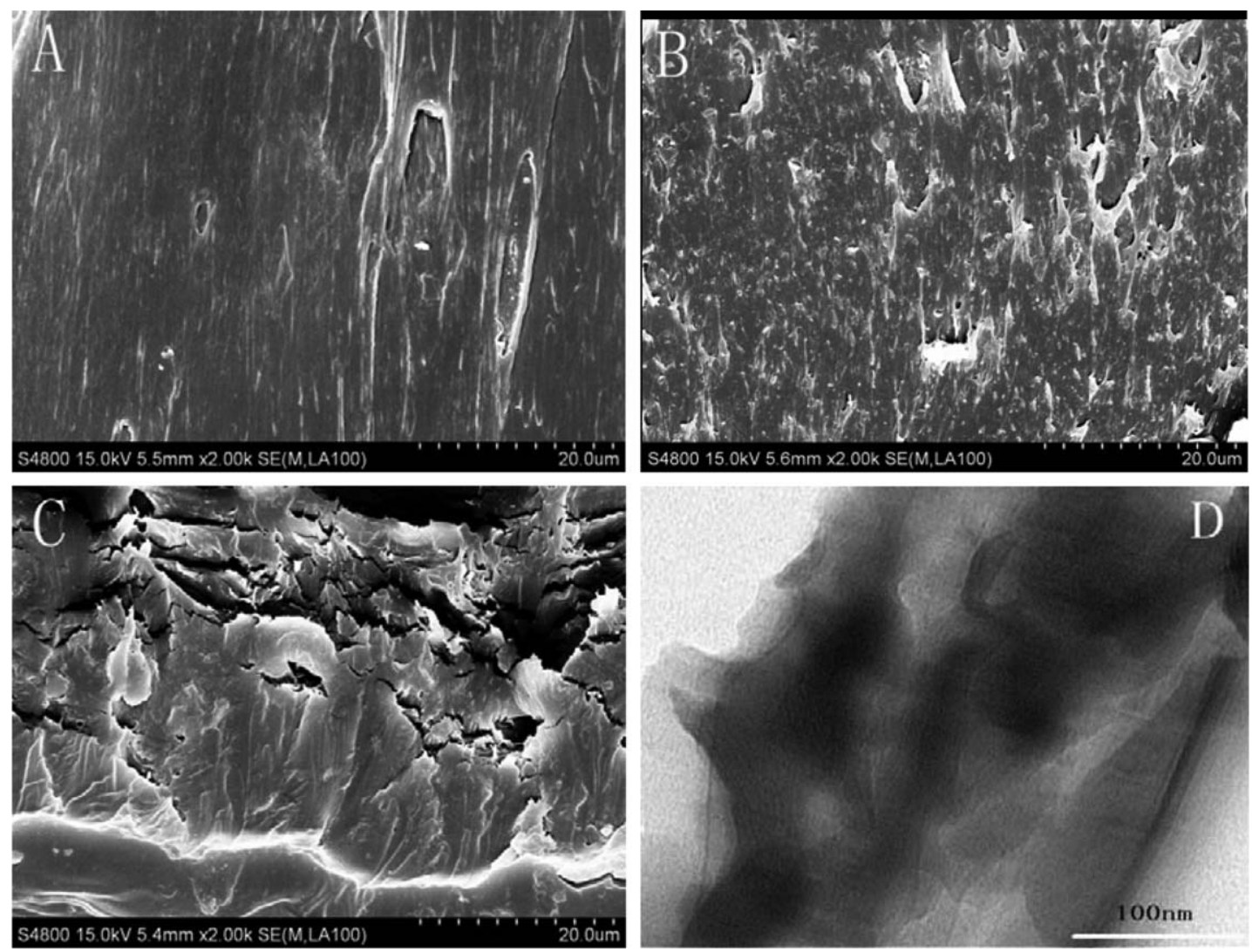

FIG. 3. SEM images of PLA/GSs-0.1 (A), PLA/GSs-1.0 (B), and PLA/GSs-2.0 (C). TEM image of PLA/ GSs-0.1 (D).

leading to less polymer cladding on the surface and weakened interfacial action, Consequently, the section roughness of the nanocomposites increases gradually. TEM image (Fig. 3D) reveals that GSs maintain the inherent layered structure after being blended with PLA, and no obvious re-aggregation is observed.

In order to confirm that the GSs in the polymer were not re-stacked, XRD measurements of pure PLA and PLA/GSs were carried out and the results are shown in Fig. 4. The XRD pattern of PLA/GSs-0.1 is almost the same as that of pure PLA, in which two characteristic diffraction peaks are shown at 16.3 and $18.7^{\circ}$, corresponding to $(200) /(110)$ and (203) planes of crystalline PLA, respectively. The diffraction peak at $26^{\circ}$ corresponding to graphite layer structure (002) is not observed in GSs due to the exfoliation. The scattering intensity profile does not reveal the presence of the diffraction peak of graphite, suggesting that the GSs do not aggregate in PLA matrix $[36,37]$. As suggested by Ramanathan et al., this result indicates that the dispersion of GSs in PLA matrix is close to single-sheet level [37].

Recently, some researchers have demonstrated that the incorporation of graphenes could dramatically enhance the thermal stability of PLA at extremely low filling content $[37,38]$. It is seen from Fig. 5A that the incorporation of GSs into PLA makes the decomposition temperature of PLA moving to higher position compared with that of
PLA. With 0.01 wt $\%$ GSs, the temperature at $5 \%$ weight loss of PLA increases about $20^{\circ} \mathrm{C}$. When the content of GSs achieves $2 \mathrm{wt} \%$, the temperature at $5 \%$ weight loss of PLA increases by more than $150^{\circ} \mathrm{C}$ (Table 2). Differential thermal gravity (DTG) curves (Fig. 5B) of pure PLA and PLA/GSs show that the weight loss rate of PLA/GSs

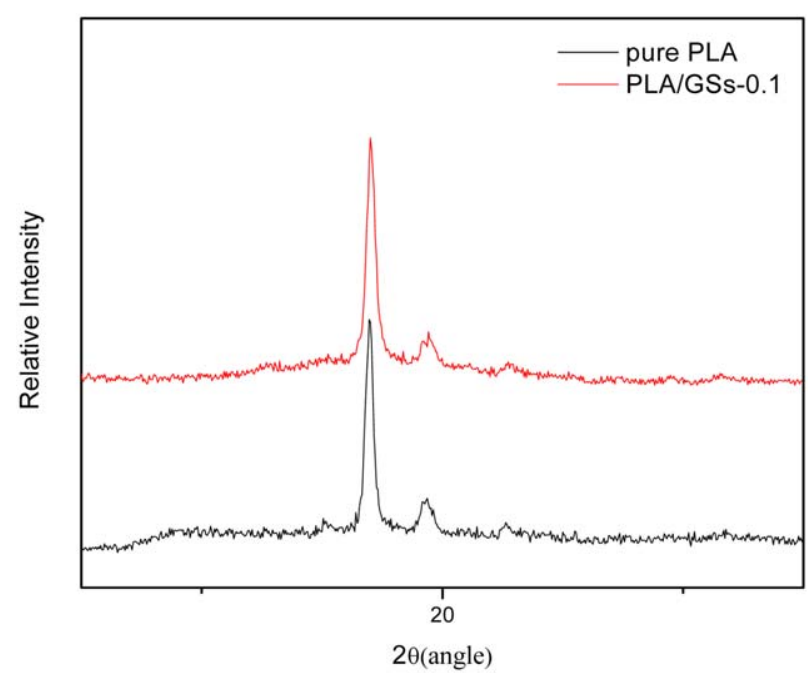

FIG. 4. XRD spectra of pure PLA and PLA/GSs nanocomposites. [Color figure can be viewed in the online issue, which is available at wileyonlinelibrary.com.] 

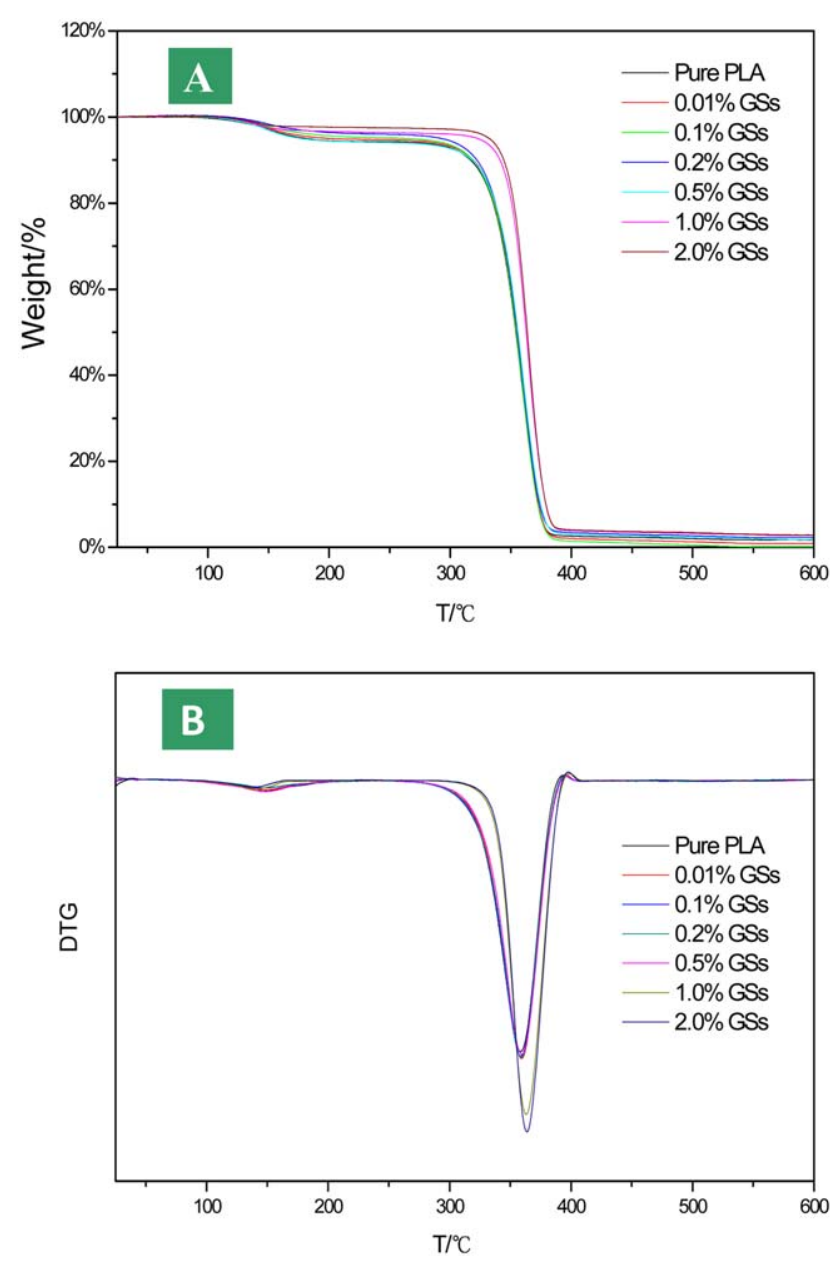

FIG. 5. TGA curves of pure PLA and PLA/GSs nanocomposites (A). DTG curves of pure PLA and PLA/GSs nanocomposites (B). [Color figure can be viewed in the online issue, which is available at wileyonlinelibrary.com.]

nanocomposites gradually decrease with the increase of GSs, especially in the early stages of the decomposition process. The improvement of thermal stability may be due to the strong polymer-GSs interfacial action, which was confirmed by SEM and TEM in the preceding part. Due to the tremendous interfacial area between GSs and PLA, the resultant radicals from PLA thermal degradation would bind with the $\pi$ conjugation system of GSs, and thus form inactive radicals to prevent further degradation, leading to lower initial decomposition rate. On the other hand, GSs

TABLE 2. TGA data of pure PLA and PLA/GSs nanocomposites.

\begin{tabular}{lccc}
\hline Sample & $T_{5 \%}\left({ }^{\circ} \mathrm{C}\right)$ & $T_{50 \%}\left({ }^{\circ} \mathrm{C}\right)$ & $T_{95 \%}\left({ }^{\circ} \mathrm{C}\right)$ \\
\hline Pure PLA & 181.13 & 354.08 & 377.48 \\
$0.01 \%$ GSs & 200.51 & 355.08 & 377.67 \\
$0.1 \%$ GSs & 263.77 & 354.46 & 376.90 \\
$0.2 \%$ GSs & 292.71 & 355.32 & 380.35 \\
$0.5 \%$ GSs & 306.63 & 355.79 & 380.29 \\
$1 \%$ GSs & 322.83 & 362.59 & 385.08 \\
$2 \%$ GSs & 332.11 & 363.51 & 385.19 \\
\hline
\end{tabular}
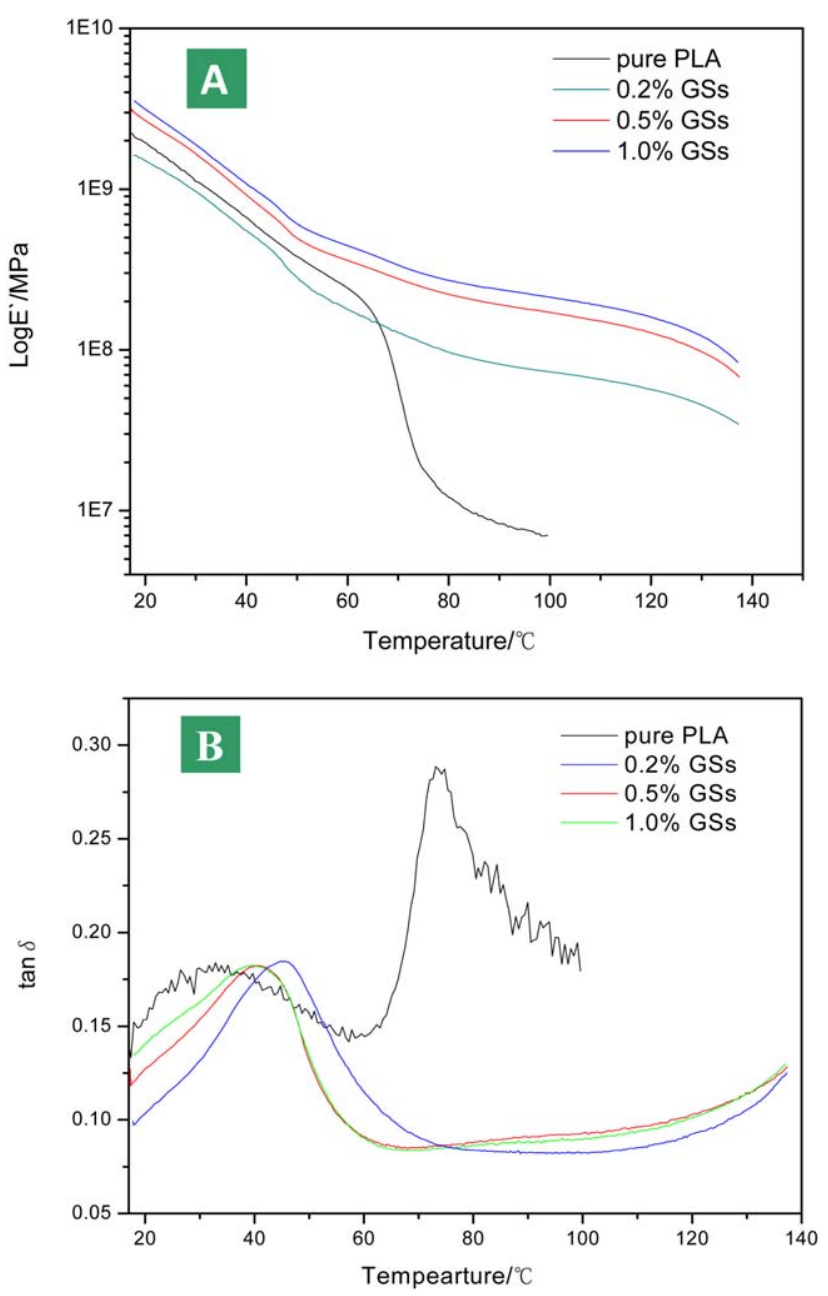

FIG. 6. Dynamic mechanical properties of PLA and PLA/GSs as a function of temperature. (A) Storage modulus $\log E^{\prime}$ and (B) damping factor $\tan \delta$. [Color figure can be viewed in the online issue, which is available at wileyonlinelibrary.com.]

with large specific surface area form a lamellar structure in PLA matrix, which plays the role of barrier and protective layers. GSs delay the penetration of oxygen and the escape of volatile degradation products, thus consequently boosts char formation.

Figure 6A shows the spectra of dynamic storage modulus $\left(E^{\prime}\right)$ as a function of temperature for neat PLA and PLA/GSs. $E^{\prime}$ is applied as a parameter of rigidity of materials. In the curve for pure PLA, two peaks of modulus loss, at 31 and $75^{\circ} \mathrm{C}$, were observed. It is apparent that the first modulus loss is due to the minor changes of PLA ( $\beta$ changes), caused by the crank movement of side chain, end group, and bond angle of polymer chain. The second loss is due to the glass transition of PLA, which is in good agreement with the data released by NatureWorks ${ }^{\circledR}$. The addition of GSs increases the $E^{\prime}$ of PLA/GSs nanocomposites within the test temperature range, especially within high temperature range. The $E^{\prime}$ of PLA/GSs does not have any obvious decrease during PLA glass transition process and increases from 6.87 

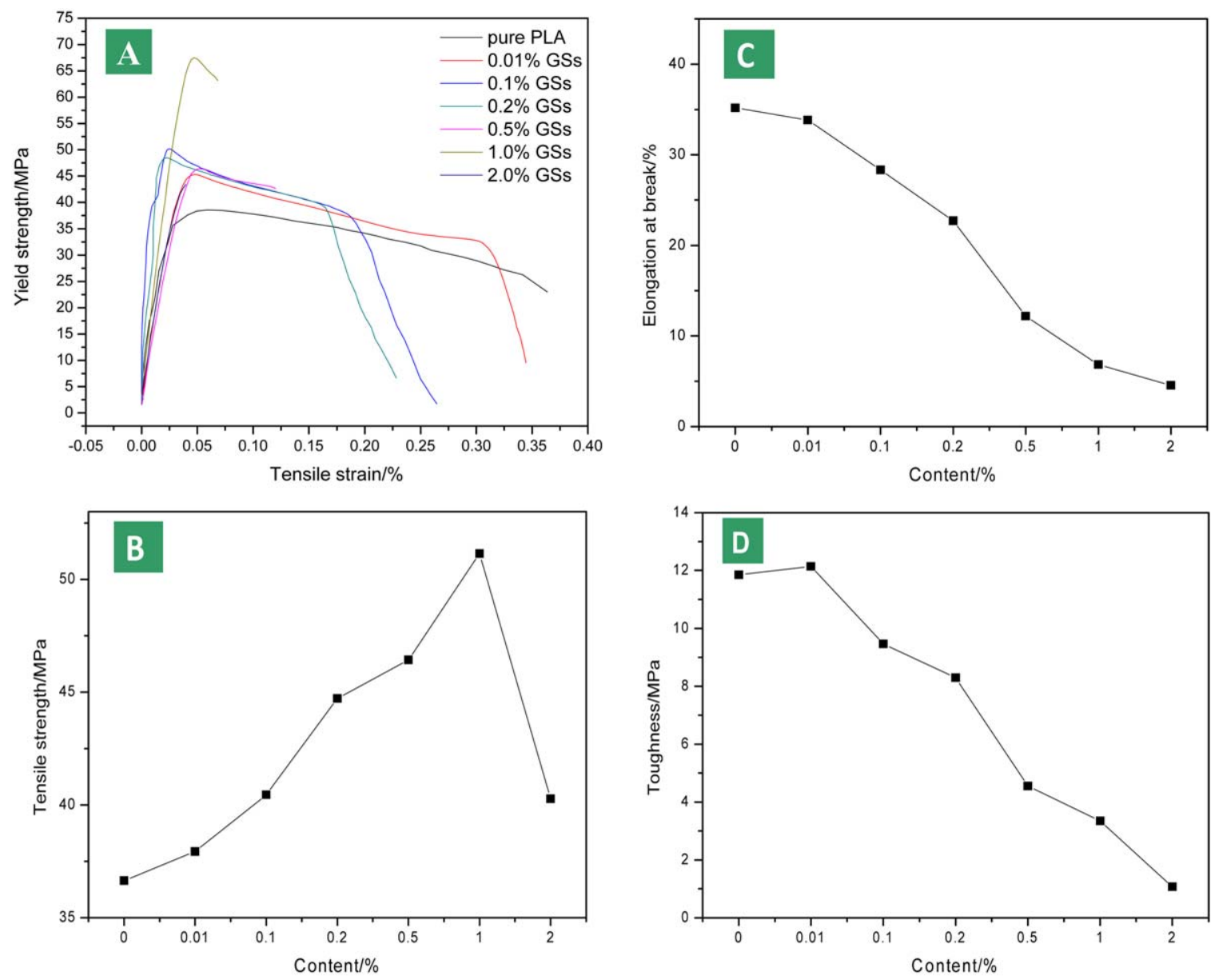

FIG. 7. Stress-strain curves (A), tensile strength (B), elongation at break (C), and toughness (D) of PLA/ GSs as a function of GS content. [Color figure can be viewed in the online issue, which is available at wileyonlinelibrary.com.]

(pure PLA) to $212.15 \mathrm{MPa}$ (PLA/GSs-1.0) at $100^{\circ} \mathrm{C}$, about 30 orders of magnitude higher compared to that of pure PLA. The phenomenon can be explained as follows. GSs, with a tremendous surface area, form a special spatial network structure in PLA matrix, leading to a strong interfacial reaction between matrix and fillers $[39,40]$. Since GSs are stiffer material than PLA, the presence of GSs produces a more rigid interface in PLA matrix [41, 42] and restricts the movement of polymer chains [43], resulting in greatly enhanced modulus of PLA matrix at high temperatures. Therefore, a GSs filled PLA composite with higher modulus at higher temperatures can be industrially manufactured. The temperature dependence of $\tan \delta$ for neat PLA and PLA/GSs is shown in Fig. 6B. The peak glass transition temperature of PLA/GSs is not observed before the peak of pure PLA $\left(75^{\circ} \mathrm{C}\right)$.

Tensile strength of PLA/GSs composite films with different GSs contents were examined at room temperature. Figure 7A shows the typical stress-strain curves of PLA/GSs and Fig. 7B shows changes of tensile strength of PLA/GSs as a function of GS content. As shown in
Fig. 7B, an increase in GSs loading from 0 to $1.0 \mathrm{wt} \%$ leads to a continuous increase of tensile strength. PLA/ GSs-1.0 has the improved tensile strength of $51.14 \mathrm{MPa}$, compared to the unreinforced matrix $(36.64 \mathrm{MPa})$. The improvement ratio is as high as $39.57 \%$. It is reasonable to conclude that the noticeable improvement of mechanical properties for PLA/GSs is attributed to the reinforcement effect of GSs dispersing homogeneously in PLA matrix. Further increment of GS loading to $2.0 \mathrm{wt} \%$ results in a decrease in tensile strength instead. This can be explained by the reduced distance between GSs (refer to Fig. 3C), which would cause highly intensified stress field between the closely spaced layers [44] and increased free volume [45]. Figure 7C exhibits the variation of the elongation at break with GS content in PLA/ GSs. It can be seen that the elongation decreases with increasing GSs content, consistent with those reported in literatures $[46,47]$. It can be explained by the fact that the strong interaction between GSs and PLA restricts the movement of the polymer chains. Figure 7D reveals the changes of PLA/GSs toughness as a function of GSs content. The toughness can be calculated from the area 
under the stress-strain curve. The toughness of the composite, similar to its elongation at break, decreases with the addition of GSs. There is a trade-off between strengthening and toughening and a great challenge remains to realize simultaneous strength and toughness enhancement.

\section{CONCLUSION}

In summary, we reported a facile and low-cost route for the preparation of PLA-based nanocomposites containing GSs to improve properties for long-term applications of degradable PLA. By this strategy, complicated oxidation-reduction process of conventional method for the fabrication of GSs can be avoided and the PLA/GSs nanocomposites can be obtained via a facile solvent blending of PLA with GS suspensions. The resultant GSs are highly exfoliated; thus the as-made dispersions of GSs in PLA are quite suitable for deposition of films. The addition of GSs in PLA greatly improves the thermal stability of the nanocomposites. The temperature at $5 \%$ weight loss increases by more than $150^{\circ} \mathrm{C}$ when GSs content achieves $2.0 \mathrm{wt} \%$. More important, the tensile strength of PLA/GSs nanocomposites is much higher than that of pure PLA, which increases from 36.64 for pure PLA up to $51.14 \mathrm{MPa}$ for PLA/GSs-1.0 nanocomposite.

\section{REFERENCES}

1. Q.K. Meng, M.C. Heuzey, and P.J. Carreau, Int. Polym. Proc., 27, 5 (2012).

2. Q.K. Meng, M.C. Heuzey, and P.J. Carreau, Polym. Degrad. Stab., 97, 10 (2012).

3. I.H. Kim and Y.G. Jeong, J. Polym. Sci. Part B: Polym. Phys., 48, 8 (2010).

4. E.E.M. Ahmad and A.S. Luyt, Polym. Compos., 33, 6 (2012).

5. D.K. Platt, Biodegradable Polymers Market report, Smithers Rapra Limited, Shawbury, Shrewsbury, Shropshire, Limited, UK (2006).

6. R.E. Drumright, P.R. Gruber, and D.E. Henton, Adv. Mater., 12, 23 (2000).

7. P.H. Dubois and M. Murariu, J. Electroanal. Chem., 45, 66 (2008).

8. E.T.H. Vink, K.R. Rábago, D.A. Glassner, and P.R. Gruber, Polym. Degrad. Stab., 80, 3 (2003).

9. S. Boncel, M. Brzezinski, and J. Mrowiec-Bialon, Mater. Lett., 91, 50 (2013).

10. M. Brzezinski, M. Boguslawska, and M. Ilcikova, Macromolecules, 45, 21 (2012).

11. H. Quan, S.J. Zhang, and J.L. Qiao, Polymer, 53, 20 (2012).

12. H.H. Ge, F. Yang, and Y.P. Hao, J. Appl. Polym. Sci., 127, 4 (2013).

13. G. Stoclet, R. Seguela, and J.M. Lefebvre, Macromolecules, 43, 3 (2010).
14. B.J. Jeon, Y.G. Jeonq, B.G. Min, W.S. Lyoo, and S.C. Lee, Polym. Compos., 32, 9 (2011).

15. M.P. Artur, C. Joana, A.P.T. David, M.M. Adelio, and D.M. Fern ao, Polym. Int., 62, 1 (2012).

16. A.M. Pinto, M. Susana, I. C. Goncalves, F.M. Gama, A.M. Mendes, and F.D. Magalhães, Colloids Surf. B., 104, 229 (2013).

17. Y.W. Cao, J.C. Feng, and P.Y. Wu, Carbon, 48, 13 (2010).

18. C.G. Lee, X.D. Wei, J.W. Kysar, and J. Hone, Science, 321, 5887 (2008).

19. A.A. Balandin, S. Ghosh, W.Z. Bao, I. Calizo, D. Teweldebrhan, F. Miao, and C.N. Lau, Nano Lett., 8, 3 (2008).

20. H.K. Chae, D.Y. Siberio-Pérez, J. Kim, Y. Go, M. Eddaoudi, A.J. Matzger, M. O'keeffe, and O.M. Yaghi, Nature, 427, 6974 (2004).

21. D.H. Youn, Y.J. Yu, and H.K. Choi, S.H. Kim, S.Y. Choi, and C.G. Choi, Nanotechnology, 24, 7 (2013).

22. C. Courgneau, S. Domenek, R. Lebossé, A. Guinault, L. Avérous, and V. Ducruet, Polym. Int., 61, 2 (2012).

23. Y.X. Kong, J. Yuan, Z.M. Wang, and J Qiu, Ploym. Compos., 33, 9 (2012).

24. L. Dan and B. Richard, Kaner, Mater. Sci., 320, 1170 (2008).

25. K.C. Kwon, K.S. Choi, B.J. Kim, J.L. Lee, and S.Y. Kim, J. Phys. Chem. C, 116, 50 (2012).

26. K.E. Carr, Carbon, 8, 155 (1970).

27. G.H. Chen, D.J. Wu, W.U. Weng, and C.L. Wu, Carbon, 41, 3 (2003).

28. W.G. Zheng, S.C. Wong, and H.J. Sue, Polymer, 43, 25 (2002).

29. Y.X. Pan, Z.Z. Yu, Y.C. Ou, and G.H. Hu, J. Polym. Sci. Part B: Polym. Phys., 38, 12 (2000).

30. E.Y. Choi, W.S. Choi, Y.B. Lee, and Y.Y. Noh, Nanotechnology, 22, 36 (2011).

31. U. Khan, H. Porwal, A. O’Neill, K. Nawaz, P. May, and J.N. Coleman, Langmuir, 27, 15 (2011).

32. A.O. Neill, U. Khan, P.N. Nirmalraj, J. Boland, and J.N. Coleman, J. Phys. Chem., 115, 13 (2011).

33. W. Liu and J. Wang, Chem. Commun., 47, 24 (2011).

34. L. Zhu, X. Zhao, Y. Li, X. Yu, C. Li, and Q. Zhang, Mater. Chem. Phys., 137, 984 (2013).

35. K.H. Park, B.H. Kim, H.S. Song, J. Kwon, B.S. Kong, K. Kang, and S. Jeon, Nano Lett., 12, 2871 (2012).

36. Y.K. Yang, C.E. He, R.G. Peng, A. Baji, X.S. Du, Y.L. Huang, X.L. Xie, and Y.W. Mai, J. Mater. Chem., 22, 12 (2012).

37. T. Ramanathan, A.A. Abdala, S. Stankovich, D.A. Dikin, M. Herrera Alonso, R.D. Piner, D.H. Adamson, H.C. Schniepp, X. Chen, R.S. Ruoff, S.T. Nguyen, I.A. Aksay, R. K. Prud'Homme, and L.C. Brinson, Nat. Nanotechnol., 3, 6 (2008).

38. S. Villar-Rodil, J.I. Paredes, A. Martinez-Alonso, and J.M.D. Tascon, J. Mater. Chem., 19, 22 (2009).

39. A.K. Rana, B.C. Mitra, and A.N. Banerjee, J. Appl. Polym. Sci., 71, 4 (1999).

40. M.S. Huda, L.T. Drzala, A.K. Mohanty, and M. Misra, Compos. Sci. Technol., 66, 11 (2006).

41. M.S. Huda, L.T. Drzala, A.K. Mohanty, and M. Misra, Compos. B, 38, 3 (2007). 
42. S.N. Nazhat, M. Kelloma, P. Tormal, K.E. Tanner, and W. Bonfield, J. Biomed. Mater. Res., 58, 4 (2001).

43. X. Zhao, Q.H. Zhang, D.J. Chen, and P. Lu, Macromolecules, 43, 5 (2010).

44. D. Broek, Elementary Engineering Fracture Mechanics, Kluwer Academic Publishers, Dordrecht, The Netherlands (1986).
45. L. An, Y.Z. Pan, X.W. Shen, H.B. Lu, and Y.L. Yang, J. Mater. Chem., 18, 4928 (2008).

46. K. Nawaza, U. Khanb, N. Ul-Haqa, P. Mayb, A. O’Neillb, and J.N. Colemanb, Carbon, 50, 12 (2012).

47. C.F. Fabiana, R.S.B. Nara, P.G. Ana, S.A. Denise, and B.G. Griselda, J. Appl. Polym. Sci., 128, 5 (2012). 